\title{
Contribución para el conocimiento del desarrollo actual de la Antropología Forense en Portugal.
}

A contribution to the state of knowledge of nowadays Forensic Anthropology in Portugal.

\section{E. Cunha 1}

RESUMEN

Se intenta dar a conocer el panorama actual de la Antropología Forense en Portugal a través de su encuadramiento en el Instituto Nacional de Medicina Legal y de su relación con otras disciplinas forenses. La docencia, el estado actual de las investigaciones sobre Antropología Forense y las publicaciones que han sido efectuadas en la última década son abordados. Se analiza el tipo, frecuencia y perfil de los casos de antropología y se concluye con ejemplos de dos casos que ilustran los procedimientos y metodologías adoptadas en Portugal.

Palabras clave: Portugal, antropología forense, esqueleto.

Cuad Med Forense 2008; 14(53-54):201-211

\section{ABSTRACT}

A state of knowledge of nowadays Forensic Anthropology in Portugal is done by means of its contextualization within the National Institute of Forensic Medicine and its relation with other forensic sciences. Teaching, research on Forensic Anthropology and the publications that had been done during the last decade are commented.

The type, frequency and profile of forensic anthropology cases in Portugal are discussed. Two cases are briefly presented in order to illustrate the procedures and methodologies adopted in our country.

Key words: Portugal, forensic anthropology, skeleton.

Correspondencia: Dra. Eugénia Cunha. Dep. de Antropología. Universidade de Coimbra. Centro de Ciências Forenses. Coimbra, Portugal. E-mail: cunhae@ci.uc.pt.

1 Profesora Caredrática de Antropología. Consultora Nacional para la Antropología Forense del INML de Portugal. 


\section{PANORAMA ORGANIZATIVO SOBRE ANTROPOLOGÍA FORENSE EN PORTUGAL:}

La Antropología Forense, tal y como hoy se conoce, tardó mucho tiempo en practicarse en Portugal. En una dimensión nacional, solamente a finales de los años noventa (1997), los peritajes del antropólogo forense empezaron a hacerse de un modo sistemático. Hasta entonces, los casos de restos esqueletizados eran hechos por los médicos en los Institutos Médico Forenses de las distintas zonas geográficas, como es el caso de Lisboa.

Para contextualizar la disciplina de Antropología Forense en Portugal, es necesario conocer la organización Médico-Forense portuguesa. El Instituto Nacional de Medicina Legal (INML) es un organismo administrativamente autónomo, que pertenece al Ministerio de Justicia. Su domicilio esta en Coimbra, en el centro del país. Hay tres delegaciones nacionales: Sur (Lisboa), Centro (Coimbra) y Norte (Porto) y 3 I gabinetes médico forenses ubicados por todo el país (Vieira, 2008). En cada delegación principal hay varios departamentos forenses: patología, toxicología, genética y clínica. La Antropología está ubicada en el Servicio de Patología Forense, es decir, que no hay en todo el Instituto portugués una unidad autónoma de Antropología Forense.

Cuando hay un caso de Antropología, como veremos posteriormente en dos ejemplos, éste es estudiado en las Delegaciones principales. La autora del presente artículo, con una formación académica en Antropología Física y Biología, es consultora nacional para la Antropología Forense en el Instituto Nacional de Medicina Forense desde 1997. Además, desde 2004, es también la responsable de todos los casos de Antropología en la Delegación del Sur (Lisboa), donde hay un gabinete propio.

Hay que añadir la participación reciente de antropólogos forenses portugueses en el caso de la Liga de los Combatientes Portuguesa, denominada "Conservación de la memoria", a través de la exhumación y posterior identificación de los cuerpos esqueletizados de los soldados muertos en la guerra colonial de Guinea-Bissau en los años 60 y 70 (dos misiones en el año 2008). Otra importante participación en el extranjero, ahora en el ámbito de Violación de Derechos Humanos, es la misión coordinada por M.C. Mendonça en Chile desde 2006: "Detenidos desaparecidos del Pateo 29, Santiago". En lo que respecta a desastres de masas, hay un equipo forense del INML dirigido por M.C. Mendonça que, en caso de necesidad, solicitan la actuación de los antropólogos.

\section{LA ENSEÑANZA DE ANTROPOLOGIA FORENSE EN PORTUGAL:}

La enseñanza de la asignatura se hace a través de cursos de postgrado y de máster (Máster en Ciencias Forenses) en los que se incluye una asignatura de Antropología Forense. Estos cursos son ofertados por las Facultades de Medicina de Lisboa, Porto y Coimbra en colaboración con el Instituto Nacional de Medicina Legal. También en la Licenciatura de Antropología del Departamento de Antropología de la Facultad de Ciencias de la Universidad de Coimbra se imparte la asignatura de Introducción a la Antropología Forense. Ésta es, además, la única Universidad y Facultad de todo el país donde se puede estudiar un tercer ciclo (doctorado) en Antropología Forense.

Lo cierto es que la Antropología Forense ha adquirido en los últimos años un mayor protagonismo en nuestro país gracias al impulso de los planes de formación que se han establecido tanto en el ámbito universitario como en el judicial.

En cualquier caso, siguiendo la nomenclatura internacional de la UNESCO, la "Antropología Forense" está considerada como una subdisciplina (2402.03) de la "Antropología Física" (2402) del campo "Ciencias de la vida" (Etxeberria, s.d.). 


\section{ESTADO ACTUAL DE LAS INVESTIGACIONES SOBRE ANTROPOLOGÍA FORENSE EN PORTUGAL:}

Muy recientemente, en 2008, se ha creado un Centro de Investigación de Ciencias Forenses que incluye una unidad de Antropología Forense con la que se espera contribuir al desarrollo de proyectos e investigaciones. La Antropología Forense portuguesa está muy implicada en el desarrollo de la disciplina en el contexto europeo, siendo muy activa en la FASE (Forensic Anthropology Society of Europe), a través de la organización de cursos, la estandarización de las metodologías y publicaciones (Cunha y Cattaneo, 2007). De este modo se celebran, desde hace unos años, "Advanced courses" o "workshops" sobre temas generales y específicos de la Antropología. También hay que hablar de la fuerte relación de la Antropología portuguesa con la ALAF (Asociación Latino Americana de Antropología Forense) desde su formación.

Además, a raíz de los cursos de Máster arriba referidos, hay ya un conjunto de tesis concluidas sobre temas que van desde la estimación de la estatura (Santos, 2004, Fuzinato, 2006) al abordaje de las afinidades poblacionales (Vilhena, 2006, Tavares, 2008), estimación de edad de los menores indocumentados (Santos, 2007; Tomaz, 2004; Julio, 2007) o determinación del sexo (Azevedo, 2008) entre otras. Muchas de estas tesis aprovechan el enorme potencial científico de las colecciones osteológicas identificadas del Museo Antropológico de la Universidad de Coimbra (Cunha y Wasterlain, 2007). Para 2009 se esperan las primeras tesis dentro del Doctorado en Antropología Forense, principalmente centradas en la reconstrucción facial (Codinha, 2009).

\section{SOBRE LOS RECURSOS BIBLIOGRÁFICOS:}

En lo que respecta a las publicaciones en Antropología hechas por autores portugueses, no son todavía abundantes pero la tendencia muestra claramente un aumento significativo en los últimos años. En 2006, la autora coeditó un libro en Humana Press: "Forensic Anthropology and Medicine, from recovery to cause of death" (Schmitt et al., 2006). Hay artículos científicos escritos por portugueses en las principales revistas internacionales de la disciplina, a decir: International Journal of Legal Medicine (Cunha et al., 2007); Forensic Science International (Cameriere et al., 2009; Codinha, 2009; Cardoso, 2008); Journal of Forensic Sciences (Schmitt et al., 2003; Rissech et al, 2006; Rissech et al., 2007; Cardoso, 2009). Por otro lado, el esfuerzo por participar en congresos internacionales de la especialidad se traduce en la presencia de antropólogos de nuestro país en varios de ellos (Cunha, 200 I; Cunha et al., 2006, Cunha et al, 2007, Mendonça, 2000; Cunha y Mendonça, 2002; Pinheiro et al., 2005; Cunha et al, 2005; Cunha et al, 2008). En la única revista sobre Antropología General editada en Portugal, la "Antropologia Portuguesa", hay artículos puntuales sobre el tema (Cunha y Pinheiro, 2006; Rissech et al, 2005).

Hay que añadir la participación en libros internacionales sobre la disciplina, a través de capítulos, de los que son ejemplo: "Forensic Anthropology, case studies from Europe" (Brickley y Ferllini, 2007) y "The world handbook of forensic anthropology and archaeology" (Blau y Ubelaker, 2009).

\section{TIPOLOGÍA DE LOS CASOS:}

Los casos son remitidos al Instituto por la Polić́a Judicial, por el Ministerio Publico o por la Guardia Civil (GNR), entre otros. Son acompañados de un breve informe sobre las circunstancias de la recogida de los hallazgos. Las entidades oficiales, como la Policía Científica, debido a una formación cada vez más adecuada, están hoy más habilitados para proceder a la recogida de los restos y a la investigación de la escena del crimen. Por otro lado, y lamentablemente, el antropólogo rara vez es incorporado para la recuperación de los hallazgos, a decir, en el trabajo de campo, si bien su participación sigue una tendencia creciente. 
Los casos remitidos y analizados en las delegaciones pueden ser distribuidos en 4 grandes grupos: los huesos animales, los huesos de contextos arqueológicos, los huesos humanos provenientes de disturbios en los cementerios actuales $y$, por fin, los verdaderos casos forenses. En este último grupo se incluyen los casos de cuerpos encontrados en varios contextos, muchas veces lugares aislados, de homicidios (Cunha et al, 2005; Cunha et al, 2007) y de individuos desaparecidos. Es este último grupo de casos el que se va a desarrollar más en el presente artículo.

Sin embargo, los restos no humanos son aproximadamente unos 20 por ciento de los casos remitidos para la Antropología. Este porcentaje es muy similar a la estadística del F.B.I. (Grisbaumn y Ubelaker, 200I), donde la frecuencia de huesos no humanos fue del 25\% del total de casos analizados en el Smithsonian Institute en un período de 25 años.

El conjunto de estudios relacionados con disturbios en cementerios se refiere a restos inhumados en cementerios actuales que son expuestos como consecuencia de trabajos de reparaciones o bien de actos vandálicos. Esta situación es también frecuente en otros países como Canadá (Rogers, 2005).

En lo que se refiere a los casos arqueológicos, cuando el contexto de recogida es conocido, el reconocimiento de la tipología del caso resulta más sencillo. El conocimiento por parte del perito de la Antropología de las poblaciones del pasado es, en estas situaciones, de una gran ayuda. Significa esto que en muchos casos, la incorporación del antropólogo en el trabajo de campo podría ahorrar tiempo y evitar gastos innecesarios, ya que podría reconocer de inmediato los hallazgos de interés puramente arqueológico.

En cuanto al cuarto grupo, el más importante, hay dos tipos de situaciones: una cuando los restos van directamente al gabinete de Antropología -lo que ocurre, fundamentalmente, si los restos están totalmente esqueletizados-; y otra cuando los restos se envían a la sala de autopsia, muchas veces como casos de patología. Bien por tener varios estados de preservación, bien por presentar lesiones traumáticas severas, en estos casos el antropólogo es llamado a intervenir.

Otra situación de interés es la que se plantea en relación a la determinación de la edad de los menores indocumentados. Durante los años de 2003 hasta 2006 los antropólogos forenses participaban en estos exámenes. Ahora, son los odontólogos quienes trabajan para el INML en la resolución de estos casos.

\section{LA FRECUENCIA DE CASOS:}

La frecuencia de casos de Antropología Forense típica se puede considerar baja. Si atendemos como ejemplo a la Delegación Sur (Lisboa), en la cual la autora lleva trabajado los últimos 5 años de un modo consecutivo, la frecuencia anual es aproximadamente de 15-20 casos. Si se añaden a este número los casos de las Delegaciones del Centro y Norte (en este último es donde hay menos casos, llegando a I por año, o incluso cero) y de los Gabinetes Médico Forenses, quienes pueden llamar a los peritos para solucionar dichos casos o reenviarlos a las delegaciones principales, el total anual de casos nunca ha superado los 30 casos durante los últimos I I años. Este número significa que un empleo de antropólogo forense a tiempo entero no está, todavía, justificado. Actualmente, en Portugal, así como en muchos otros países europeos y no europeos, la demanda de empleo en Antropología Forense es mucho mayor que la oferta, lo que se refleja en los cursos de antropologia forense que se caracterizan por tener muchos estudiantes. 


\section{EL PERFIL DE LOS CASOS:}

Una vez excluidos los restos óseos no humanos, se pude afirmar que la pieza ósea más frecuentemente remitida es el cráneo (Manuel, 2008) "Cráneo encontrado cerca de una calle, en un lugar aislado, en la playa, etc.").

Los esqueletos completos suelen encontrarse cuando las piezas de vestuario están todavía en el cuerpo, ayudando a mantener los huesos en articulación.

Los hallazgos humanos con muchas alteraciones tafonómicas, sobre todo con raíces y huellas de dientes de animales necrófagos, son frecuentes. Los casos de cremación, con huesos carbonizados, también ocurren pero con menor frecuencia.

Tal vez, el perfil de caso más frecuente en nuestro país es el de individuos ancianos perdidos cuyos cuerpos son hallados en lugares remotos, completamente esqueletizados y todavía con la ropa puesta (Cunha y Pinheiro, 2007; Pinheiro et al, 2004; Cunha et al, 2005). El porcentaje de homicidios es relativamente bajo; hay a destacar sólo dos casos de mutilación corporal en 2007/2008.

En cuanto a la frecuencia de identificaciones positivas, desde 1997 hasta la actualidad la tendencia es creciente, fruto de un trabajo de cooperación cada vez mejor con las entidades policiales con quienes se intenta compartir el trabajo de la investigación criminal. En varios casos, la identificación positiva solamente se consiguió unos 6 meses después de enviado el informe. Normalmente se hace público el perfil biológico del individuo en la zona geográfica donde los hallazgos humanos han sido recuperados, manteniendo siempre el diálogo entre la policía y el perito. Otras veces, la lista de desaparecidos de la policía es analizada haciendo una búsqueda de individuos con un perfil compatible con el examinado. En suma, puede decirse que de los casos verdaderamente forenses (lo que en Portugal significa que la muerte ocurrió en los últimos 15 años) un tercio es identificado positivamente.

\section{EL EXAMEN DE LABORATORIO:}

Por lo que se ha mencionado, el trabajo del antropólogo empieza, en la mayoría de los casos, en los gabinetes o en la sala de autopsia. La interpretación tafonómica es la primera fase del peritaje en el cual pueden entrar otros peritos como el entomólogo o el botánico. Todas las alteraciones cromáticas, de destrucción post-mortem, marcas/huellas de animales y de plantas son anotadas. Solamente después se empieza la etapa de la limpieza de los restos humanos, que es de capital importancia (Pinheiro y Cunha, 2006). Si todavía hay tejidos blandos se procede a la maceración de los mismos. La reconstrucción ósea es efectuada siempre que sea necesario.

Cuando un mismo cuerpo presenta varios estadios de conservación, lo más frecuente es que el cráneo se encuentre esqueletizado, el tórax saponificado y las extremidades momificadas. En estos casos, y a nuestro entender, el mejor equipo de técnicos en Antropología llamados a intervenir quedaría constituido por un antropólogo forense (antropólogo físico), un patólogo forense (médico especialista en medicina legal y forense), un odontólogo forense (odontólogo) y un biólogo forense (experto en genética) (Cunha et al, 2006). En la práctica, en nuestro país, la realidad es que el patólogo hace su examen y su informe y el antropólogo el suyo independientemente. No obstante, durante la última década hay varios ejemplos de informes compartidos y firmados conjuntamente por el patólogo y por el antropólogo. Los dientes son analizados mayoritariamente por parte del antropólogo, que se encarga del odontograma y de la comparación con los registros dentarios antemortem. Las muestras de hueso son recorridas en el examen antropológico y remetidas, cuando así se necesita, al Servicio de Biología y Genética del INML. 
Las metodologías utilizadas para el análisis del esqueleto son las más actuales posibles y, siempre que las hay, las que han sido testadas o desarrolladas con base en colecciones de referencia portuguesas. La estandarización de los métodos es uno de los objetivos de la FASE y, en este sentido, procuramos aplicar las metodologías recomendadas (Cunha et al., 2008/9). Como ejemplo, referimos aquí la estimación de la edad para la cual el método "Two steps procedure" es el utilizado (Baccino y Schmitt, 2006). El método Suchey-Brooks es aplicado a la sínfisis púbica, combinado con el método de Lamendin (Lamendin et al., 1993) que evalúa la transparencia radicular (es uno de los seleccionados para los individuos adultos). Para éstos, el cierre de la superficie esternal de la clavícula, las vértebras y las extremidades esternales de las costillas son siempre verificadas.

Lo mismo ocurre con todos los otros parámetros del perfil biológico: diagnóstico del sexo, evaluación de las afinidades poblacionales y la determinación de la estatura.

Una vez obtenido el perfil biológico, el perito hace una búsqueda de los factores/huellas de individualización del esqueleto que lo van a diferenciar de los demás. Aquí las lesiones pueden adquirir una gran relevancia (Cunha, 2006).

Para el conocimiento de la causa y circunstancias de la muerte, el papel del antropólogo consiste, por encima de todo, en interpretar las lesiones traumáticas con características perimortales. Como el certificado del óbito es emitido exclusivamente por el médico o patólogo, la discusión de esta información debe ser conjunta. En los casos portugueses, el trabajo del antropólogo ha resultado fundamental en la discriminación de las lesiones ante, peri y post mortem. Además, entre las peri-mortem, la discriminación entre lesiones contusas, cortantes o incisas (Cunha, et al., 2007).

Otro parámetro sobre el cual el antropólogo siempre resulta determinante es la data de la muerte. Lamentablemente, el desconocimiento del lugar donde los restos han sido recuperados, impide, muchas veces, hacer un juicio. De todas maneras, el antropólogo intenta lograr una estimación, aunque muy general, del periodo de tiempo transcurrido desde la muerte con bases en el estado de conservación de los restos óseos y de las prendas de vestuario que eventualmente lo acompañan.

\section{LA ELABORACIÓN DE LOS INFORMES TÉCNICOS:}

Por supuesto que de todo el proceso se deberá elaborar el correspondiente informe pericial teniendo presente la información recogida en la ficha "ante mortem" así como en el procedente del correspondiente examen de laboratorio (Etxeberria, n.d.).

Los informes deben ser tan completos como sea posible, incluyendo todos los procedimientos y resultados, y refiriendo siempre las metodologías utilizadas. Se intentará en todo caso poner las conclusiones de una forma clara y breve.

La identificación puede ser positiva (concordancia absoluta), de gran probabilidad (BTBbelieve to be, concordancia relativa) cuando hay una gran consistencia entre los datos ante y post mortem pero todavía no se puede atribuir una identidad, y no identificado (discrepancia de datos) por falta de evidencia o material insuficiente. En Portugal, la insuficiencia o la ausencia total de datos ante mortem, es el motivo más frecuente que hace no viable la identificación positiva. Como, fundamentalmente, la identificación es un proceso comparativo, la ausencia de datos para efectuar dicha comparación, imposibilita la identificación, a pesar de haber realizado un examen muy completo.

En los casos en que no se pueda identificar al fallecido, los restos exhumados y todas las pruebas son conservadas en el Gabinete de Antropología de una de las Delegaciones del INML, para garantizar la cadena de custodia. 


\section{EJEMPLOS DE CASOS:}

Con el fin de ejemplificar los procedimientos anteriormente anotados se reproducen aquí dos casos de rutina de Antropología Forense ya concluidos, llevados a cabo en la Delegación Sur del INML. En efecto, entre las decenas de casos investigados por la autora, se presentan dos historias que muestran cómo los muertos pueden hablar.

\section{CASO I}

En el primero, un conjunto de huesos humanos pertenecientes al mismo cuerpo fue recuperado en un lugar aislado de una sierra en las cercanías de Lisboa. Una vez colocados los huesos en articulación, se apreció que el esqueleto estaba incompleto, sobre todo en lo que respecta al esqueleto axial. Además, los huesos se encontraban recubiertos de cal, lo que les daba un intenso color blanco. El perfil biológico fue establecido: se trataba de una adolescente con unos 17-20 años de edad, caucásica y de estatura mediana. En las epífisis distales de la tibia izquierda, así como en el astrágalo del mismo lado, había fracturas, algo sutiles, con características peri mortales que apuntaban a una caída desde un lugar alto, con aterrizaje sobre los pies (Fig. I). Unos meses después de entregar el informe, se encontró una desaparecida con el mismo perfil biológico. La adolescente estaba desaparecida desde hacía varios meses y su familia no podía creer que su cuerpo estuviera ya esqueletizado. Así, con la concordancia de los datos, se comenzó a sospechar que podría ser. Entre tanto, el antropólogo insistió a las autoridades para volver al lugar de los hallazgos e intentar recoger el resto de los huesos. La policía, una vez en el lugar, encontró algunos huesos más y objetos personales. Además, se apreciaba una cantidad significativa de cal sobre los huesos. Una vez en el laboratorio, se verificó que los huesos recuperados pertenecían al mismo esqueleto. Más importante, la familia reconoció los objetos personales. A través del análisis genético, la identificación fue confirmada y el cuerpo devuelto a la familia. La sospecha de homicidio se enfatizó tanto por la fractura en la epífisis distal de la tibia y superficie articular superior del astrágalo, como por las circunstancias de los hallazgos, recubiertos de cal que parecía puesta directamente en varias ocasiones sobre los huesos intentando cubrirlos o hacerlos desaparecer. No fue posible encontrar al autor y la hipótesis relativa a la etiología

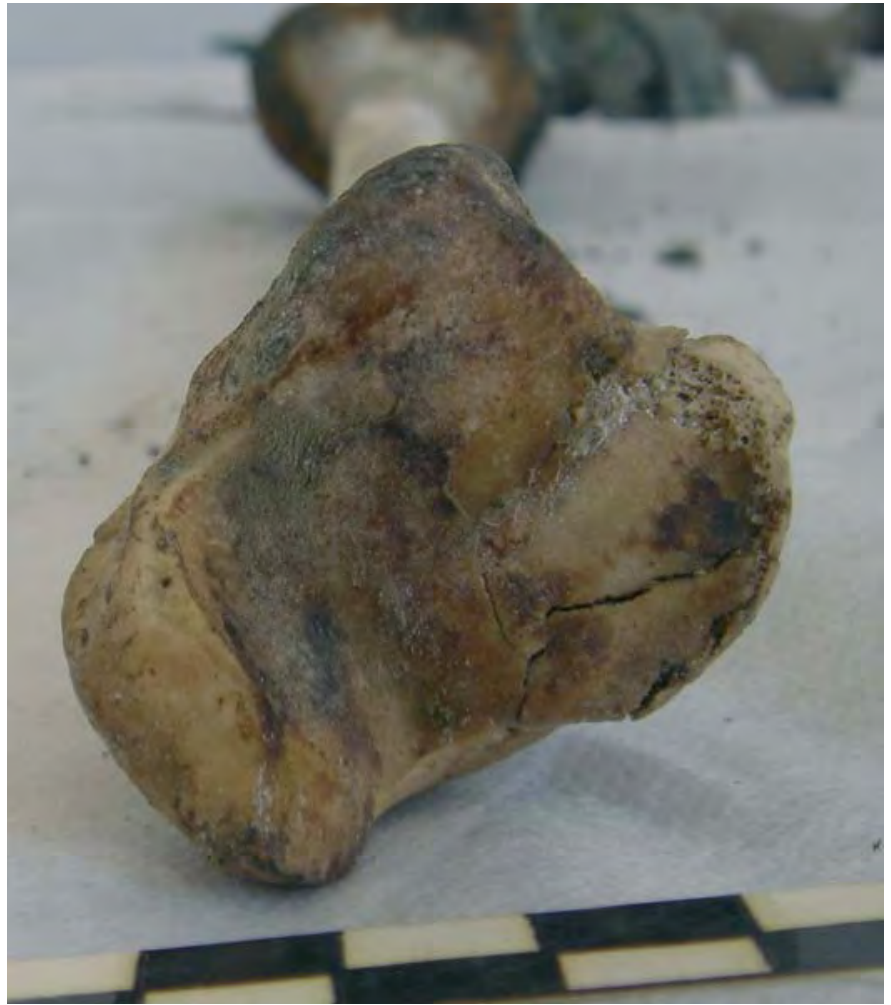

Figura 1.- Fractura peri mortal en la epífisis distal de la tibia, en la superficie articular inferior, compatible con una caída de un lugar alto. Cortesía del INML de Portugal. 
médico-legal de homicidio no pudo confirmarse (la hipótesis de suicidio tampoco pudo ser descartada en absoluto). Solamente se pudo decir que se trató de una muerte violenta.

En conclusión, en este caso la identificación positiva se consiguió unos meses después de concluido el informe. Sobre la causa de la muerte violenta, la fuerte sospecha de homicidio no ha podido ser confirmada hasta hoy.

\section{$\mathrm{CASO} 2$}

El segundo caso se refiere a un esqueleto bastante completo y parcialmente vestido que se encontró en los terrenos de una prisión portuguesa. El perfil biológico conseguido fue el de un individuo caucásico, con unos 30-45 años de edad a la muerte y de estatura mediana. El examen antropológico permitió, también, aportar datos sobre la causa de la muerte: se encontraron lesiones craneales traumáticas peri mortales compatibles con una muerte violenta (Fig. 2). En cuanto al tiempo transcurrido desde la muerte, el color blanco secundario a la exposición solar prolongada de algunos huesos, acompañado de unas fracturas longitudinales post mortem (Fig. 3) y el hecho de que todos los huesos se hallasen completamente secos, apuntaba a un período largo. Además, el análisis entomológico aportó también datos a este respecto. Los datos del informe, una vez en manos de la policía, permitieron un trabajo detenido de confrontación con la lista de desaparecidos de la prisión: unos 80 desde la fecha probable de la desaparición (en relación con el tiempo transcurrido desde la muerte calculada con bases en los hallazgos óseos y entomológicos). Tras un año de trabajo exhaustivo de las autoridades, siempre en comunicación con la experta en Antropología, se encontró la identidad de la víctima (confirmada genéticamente). Todavía, las circunstancias de la muerte no han podido ser confirmadas y, hasta hoy, esta cuestión permanece abierta.

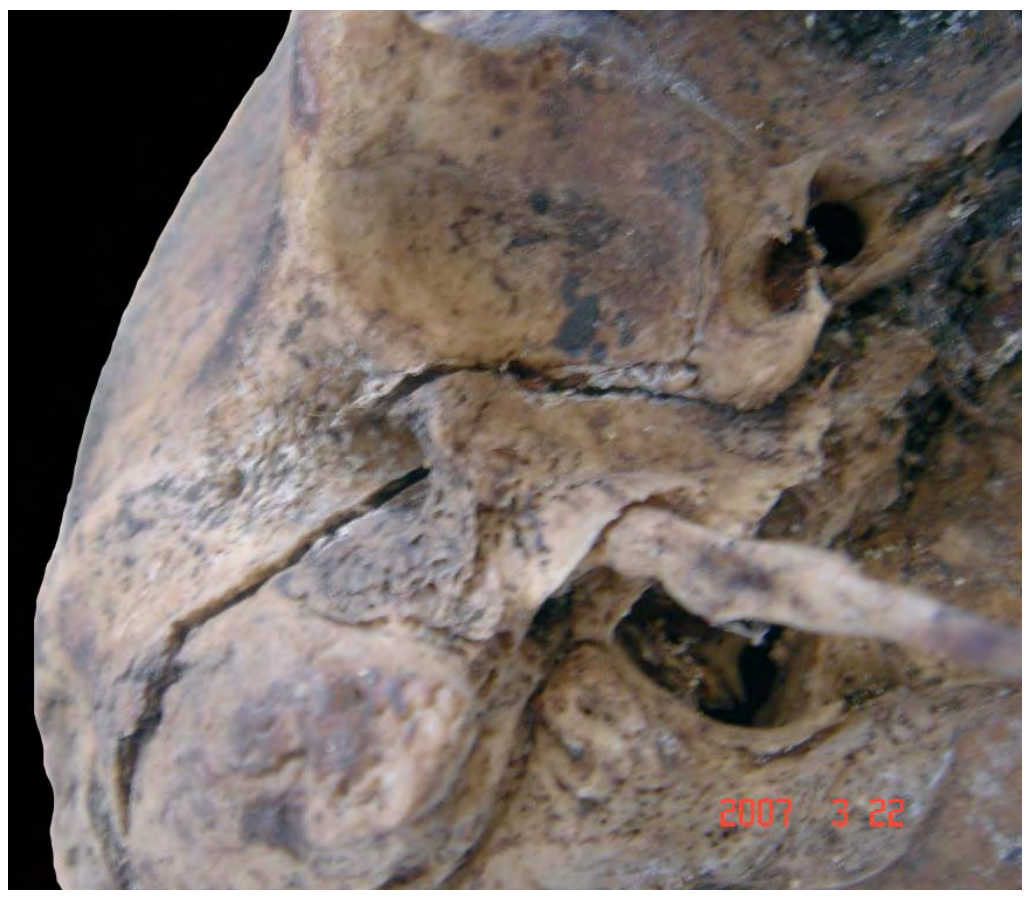

Figura 2.- Fractura craneana peri mortal en la base del cráneo compatible con una muerte violenta. Cortesía del INML de Portugal. 


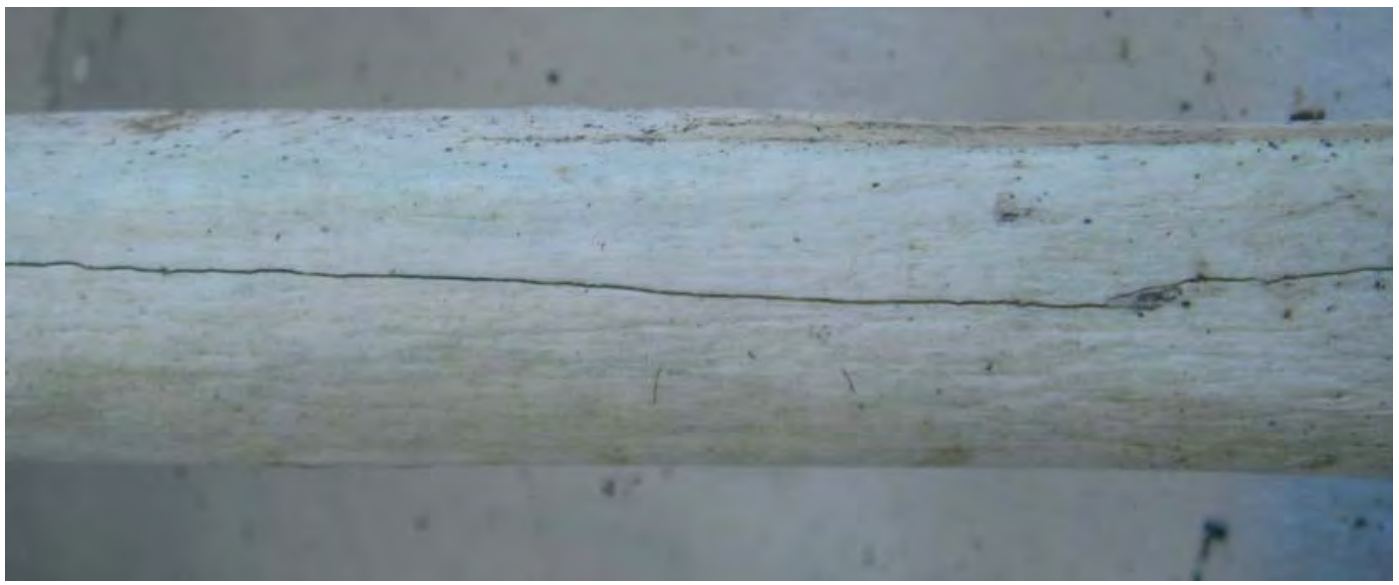

Figura 3.- Diáfisis del fémur donde se puede observar el color blanco así como la fractura longitudinal ocurrida post-mortem. Ambos efectos fueron debidos a la exposición solar prolongada. Caso 2. Cortesía del INML de Portugal.

En ambos casos fue el perfil biológico obtenido por la Antropología el que permitió diagnosticar la identidad de los fallecidos. La identificación se corroboró por la genética. En los dos casos, la muerte fue violenta pero los responsables de las mismas aún no han sido identificados.

\section{BIBLIOGRAFIA:}

- Azevedo, J. 2008. A eficácia dos métodos de diagnose sexual em antropologia forense. Mestrado em Medicina Legal e Ciências Forenses. Faculdade de Medicina. Universidade de Lisbopa. No publicada.

- Baccino, E.; Schmitt. 2006. Determination of adult age at death in Forensic Context. In: : Schmitt A., Cunha E., Pinheiro J. (eds) Forensic anthropology and Medicine: complementary sciences from recovery to cause of death. New Jersey. Humana Press:259-280.

- Blau,S.; Ubelaker, D. 2009. Handbook of Forensic Anthropology and Archaeology : Digging Deeper: Current Trends and Future Directions in Forensic Anthropology and Archaeology (WAC)Research Handbook of Forensic Anthropology and Archaeology. In press.

- Brickley,N.d Ferllini,R.(ed).2007. Forensic anthropology. Case studies from Europe. London. Charles C.Thomas.

- Cameriere, R. Cunha,E. Sassaroli E, Nuzzolese E, Ferrante 2008Age estimation by pulp/tooth area ratio in canines: study of a Portuguese sample to test Cameriere's method. Forensic Scienc Interna. Submitted.

- Cardoso, H.F. 2007. A test of the differential accuracy of the maxillary versus the mandibular dentition in age estimations of immature skeletal remains based on developing tooth length. J Forensic Sci. 2007 Mar;52(2):434-7.

- Cardoso, H.F. 2009. A test of three methods for estimating stature from immature skeletal remains using long bone lengths.J Forensic Sci. 2009 Jan;54(I):13-9. Epub 2008 Nov 21.

- Cardoso,H.F. 2007. Accuracy of developing tooth length as an estimate of age in human skeletal remains: the deciduous dentition. Forensic Sci Int. 2007 0ct 2;172(I):17-22.
- Codinha, S. 2009. Facial soft tissue thicknesses for the Portuguese adult population. Forensic Sci Int. 2009 Jan 3. [Epub ahead of print] Cordeiro, C.; Cunha, E.; Vieira, D. N. 2002. Predicting stature from metatarsal lengths. Journal de Medecine Legale Droit Medical Victimologie Dommage Corporel. N4-5.Vol.45: 128.

- Cordeiro,C.; Wasterlain,S.; Cunha, E.; Vieira,DN. 2003. How accurate is the estimation of stature from metatarsals lenghts? Forensic Science International. Vol. I36. supp.I: I64.

- Cunha, E. 200I. When forensic anthropology is disturbed by population derived assumptions. American Journal of Physical Anthropology*. Supp. 32: 56.

- Cunha, E. 2006. Pathology as a factor of individualization in forensic anthropology In: Schmitt A., Cunha E., Pinheiro J. (eds) Forensic anthropology and Medicine: complementary sciences from recovery to cause of death. New Jersey. Humana Press. 333-358.

- Cunha, E.; Mendonça, M. C. 2002. Anthropologie medico-legale: contribution de la paleopathologie a un cas des Açores. In: Temas de Medicina Legal II. Centro de Estudos de Pós-Graduação em Medicina Legal. Coimbra: I2I-I26.

- Cunha, E.; Mendonça, M. C. 2002. Forensic Anthropology in Portugal: the state of knowledge. In: Proceedings American Academy of Forensic Sciences Annual Meeting: 230.

- Cunha, E.; Mendonça,C.; Vieira,D.N. 2005.Exhumation and identification of a particular individual in a mass grave. Proceedings American Academy of Forensic Sciences. Annual Meeting. New Orleans::314.

- Cunha,E.; Pinheiro,J. 2007. A linguagem das fracturas. Sinais de violência em antropologia forense. Antropologia Portuguesa. Vol. 22:223-246. 
- Cunha, E.; Pinheiro, J. 2007 Forensic anthropology in Portugal: from current practice to future challenges. In Brickley and Ferllini (ed).Forensic anthropology. Case studies from Europe. Charles C.Thomas:38-58.

- Cunha, E.; Pinheiro, J.; Vieira, D. N. 2002. Pathology as a factor of individualization in forensic Anthropology. Journal de Medecine Legale Droit Medical victimologie Dommage Corporel. N4-5. Vol.45: 127.

- Cunha,E.; Badal,J.; Liryo,A.; Pinheiro,J.; Symes,S. 2008. How easily can we derive cause and manner of death on the basis of dry bones? Lessons derived from Coimbra Identified Skeletal Collections. Proceedings American Academy of Forensic Sciences. Washington d.c. 370.

- Cunha,E.; Cattaneo,C. 2006. Forensic Anthropology: and Forensic Pathology. The state of the art. In: Schmitt A., Cunha E., Pinheiro J. (eds) Forensic anthropology and Medicine: complementary sciences from recovery to cause of death. New Jersey. Humana Press. 39-56. - Cunha,E.; Pinheiro, J. 2009. Ante mortem trauma. In Blau,S.; Ubelaker, D. Handbook of Forensic Anthropology and Archaeology: Digging Deeper: Current Trends and Future Directions in Forensic Anthropology and Archaeology (WAC)Research Handbook of Forensic Anthropology and Archaeology. In press.

- Cunha,E.; Pinheiro,J.; Corte Real,F. 2005. Two Portuguese homicide cases: the importance of interdisciplinarity in forensic anthropology. "ERES (Arqueología y Bioantropología". Vol I3: 65-72.

- Cunha,E.; Pinheiro,J.; Corte-Real,F. 2005. When old people get lost. The identification of their bodies. 17th Meeting of the International Association of Forensic Sciences. Hong-Kong. August 2005.

- Cunha,E.; Pinheiro,J.; Ribeiro,I.P.; Vieira, D.N. 2007. Exchanged identities in a complex multiple homicide case. Part I: Identification International Journal of Legal Medicine. I2I (6):483-488.

- Cunha,E.; Pinheiro,.;; Vieira, D.N. 2006. Identification in forensic anthropology and its relation with genetics. International Congress series 1288, pp. 807-809.

- Cunha,E.; Wasterlain,S. 2007.The Coimbra Identified Skeletal collections. In Grupe, G.; Peters,J. (ed). Skeletal series and their socio-economic context. Verlag Marie Leidorf. Rahden. 23-34.

- Cunha,E. Cattaneo,C.; Baccino,E. Et al. 2008/9. A review on aging.

Forensic Science International. Submitted.

- Etxeberría, F.G. Panorama organizativo sobre Antropología y Patología Forense en España. Algunas propuestas para el estudio de fosas con restos humanos de la Guerra Civil española de 1936. ht t p : // www.sc.ehu.es/scrwwwsr/MedicinaLegal/_private/AnPat.htm.

- Fuzinato,D. 2005 Estimativa do Comprimento do Fémur e úmero a partir da medida dos seus gragmentos na população portuguesa. Masters's thesis on Medicina Legal. Faculdade de Medicina. Universidade de Coimbra. No publicado.

- Grisbaum,G.A.; Ubelaker,D.200I. An analysis of forensic anthropology cases submitted to the Smithsonian Institution by the Federal Bureau of Investigation from 1962 to 1994. Contributions to Anthropology. № 45. Smithsonian Institution Press: Washington.
- Júlio, P. 2007. Contribuição para o melhor conhecimento da idade dos menores indocumentados. Dissertação em Mestrado de Ciências Forenses. Faculdade de Medicina. Universidade do Porto. No publicado.

- Lamendin,H.; Baccino,E.; Humbert,J.; Tavernier,J.C.; Nossintchouk,R.; Zerilli,A. 1993. A simple technique for age estimation in adult corpses: the two criteriadental method. J. Forensic Sci. 37.5: 1373-9.

- Manuel, R.S. 2008: "0 perfil dos casos de antropologia forense da delegação de Lisboa do INML: contribuiç̧ão para a organização do respectivo serviço". Dissertação de Mestrado em Ciências Forenses. Faculdade de Medicina. Universidade de Lisboa. No publicado.

- Mendonça, M.C. 2000.Estimation of height from the lenght of long bones in a Portuguese adult population. Am. J.Phys. Anthropol, II2.I: 39-48.

- Pinheiro, J.; Cunha, E .;Cordeiro, C.;:; Vieira, D. N. 2004. Bridging the gap between forensic anthropology and osteoarchaeology: a case of vascular pathology. International Journal of Osteoarchaeology. Vol.I4.2:137-144.

- Pinheiro,J.; Cunha, E. 2006.Forensic Investigation of Corpses in various states of decomposition: a multidisciplinary approach. In: Schmitt A., Cunha E., Pinheiro J. (eds) Forensic anthropology and Medicine: complementary sciences from recovery to cause of death. New Jersey. Humana Press. 159-196.

- Pinheiro,J.; Cunha,E.; Cattaneo,C.; Corte-Real, F. 2005. Forensic Anthropologist and Forensic Pathologist: why work together? Proceedings American Academy of Forensic Sciences. Annual Meeting. New Orleans: pg.30l.

- Pinheiro,J.; Lyrio,A.; Cunha,E.; Symes, S. 2008. Cranial bone trauma. Misleading injuries. Proceedings American Academy of Forensic Sciences. Washington d.c.:363.

- Rissech, C.; Estabrook,G.; Cunha,E.; Malgosa, A. 2006. Using the Acetabulum to Estimate Age at Death of Adult Males. Journal of Forensic Sciences. Vol. 5I.n²: 213-229.

- Rissech,C. Estabrook,G..; Cunha, E..; Malgosa,A, 2007. Estimation of Age at Death for Adult Males Using the Acetabulum, Applied to four Western European Populations. Journal of Forensic Sciences. Jul;52(4):774-8.

- Rissech,C.; Schmitt,A.; Malgosa,A.; Cunha,E. 2005. Influencia de las patologías en los indicadores de edad adulta del coxal: estudio preliminar. Antropologia Portuguesa. vol 20/21.

- Rogers, T. 2005. Recognition of cemetery remains in a forensic context. J. For. Sci., 50 (I); 5-II.

- Rougé-Maillart, C; Jousset,V. ; Vielle,B. ; Cunha,E. ; Penneau, M. ; Telmon, N. 2007. Post mortem age estimation of male adult by the acetabulum and the auricular surface. Study from a sample of 440 bones. Journal de Medicine Légale et Droit Médicale. Vol 49. 7-8: 295- 398. - Santos, C. 2003). Estimativa da estatura com base nos metatársicos. Masters's thesis on Medicina Legal. Faculdade de Medicina. Universidade de Coimbra. No publicado. 
- Santos,C. 2007 Estimativa da idade de não-adultos( ossificação da mão).Dissertação de Mestrado em Ciências Forenses. Faculdade de Medicina. Universidade de Coimbra. No publicado.

- Schmitt A., Cunha E., Pinheiro J. (eds) 2006 Forensic anthropology and Medicine: complementary sciences from recovery to cause of death. New Jersey. Humana Press.

- Schmitt, A.; Murail, P.; Cunha, E.; Rougé, D.; 2002. Variability of the pattern of aging on the human skeleton: evidence from bone indicators and implications on age at death estimation. Journal of Forensic Science*s. Vol.47.n.6: I203-1210.

- Tavares,P.M. 2008. As afinidades Populacionais na identificação forense: métodos de avaliação e teste ao Programa Fordisc 2.0. Dissertaçãa de Mestrado em Medicina Legal e Ciências Forenses. № publicado.
- Tomás,L.F.D. 2005, Padrões de erupção e calcificação dentárias, numa amostra populacional portuguesa. Interesse médico-Legal. Dissertação de Mestrado em Medicina Legal. Faculdade de Medicina. Universidade de Coimbra. No publicada.

- Vieira, D.N. 2008. Forensic Medicine in Portugal. In Madea,B.; Saukko,P (eds). Forensic Medicine in Europe. Lubeck. Scmidt \& Ronhild: 317-34I.

- Vilhena de Carvalho, M.L. 2006. Fordisc 2.0: Nova metodologia para a Antropologia Forense Portuguesa? Dissertação de Mestrado em Medicina Legal, Universidade de Coimbra. Faculdade de Medicina. No publicado. 